\title{
Hybrid Ultrasound Tomography Scanner-a Novel Instrument Designed to Examine Breast as a Breast Cancer Screening Method
}

\author{
Milewski Tomasz ${ }^{1,2}$, Michalak Maciej ${ }^{1,2}$, Wiktorowicz Andrzej ${ }^{3}$, Opieliński Krzysztof ${ }^{4}$, Pruchnicki Pi- \\ otr $^{4}$, Bułkowski Mariusz ${ }^{3}$, Gielecki Jerzy ${ }^{5}$ and Jóźwik Marcin*6,7 \\ ${ }^{1}$ Department of Diagnostic Imaging, Independent Public Care Facility of the Ministry of the Interior and Warmian \& Mazurian Oncology \\ Center, Olsztyn, Poland \\ ${ }^{2}$ Department of Radiology, Collegium Medicum, University of Warmia and Mazury in Olsztyn, Poland \\ ${ }^{3}$ DRAMINSKI Ultrasound Scanners Company, Olsztyn, Poland
}

${ }^{4}$ Department of Acoustics and Multimedia, Faculty of Electronics, Wroclaw University of Science and Technology, Wroclaw, Poland

${ }^{5}$ Department of Anatomy, Collegium Medicum, University of Warmia and Mazury in Olsztyn, Poland

${ }^{6}$ Department of Gynecology and Obstetrics, Collegium Medicum, University of Warmia and Mazury in Olsztyn, Poland

${ }^{7}$ Gyneka Center for Woman's Health, Krakow, Poland

*Corresponding author: Jóźwik Marcin, Department of Gynecology, Gynecologic Oncology and Obstetrics, Olsztyn Municipal Hospital, Al. Niepodleglosci 44, 10-045 Olsztyn, Poland

\section{ARTICLE INFO}

Received: 慧 February 04, 2019

Published: 幽 February 15, 2019

Citation: Milewski T, Michalak M, Wiktorowicz A, Opieliński K, Pruchnicki P, Bułkowski M, Gielecki J, Jóźwik M. Hybrid Ultrasound Tomography Scanner-a Novel Instrument Designed to Examine Breast as a Breast Cancer Screening Method. Biomed J Sci \& Tech Res 14(4)-2019. BJSTR. MS.ID.002594.

\section{ABSTRACT}

Alternative radiological methods have been searched to improve early breast cancer detection. A prototype of a hybrid ultrasound tomography scanner was constructed as a novel non-invasive breast cancer screening method. The results of measurements with the new instrument were compared to conventional breast ultrasound, mammography, and magnetic resonance data from these same patients. The estimated hybrid ultrasound tomography scanner sensitivity was $74 \%$, and specificity was $68 \%$. Although these results do not match classical radiological methods yet, this preliminary report confirms the ability of the hybrid ultrasound tomography scanner to detect and visualize different types of breast lesions including breast cancer, providing a rationale for further research.

\section{Introduction}

Breast cancer is the most frequent malignancy in women worldwide. The key factor for its complete cure is early dection of the lesion. In any clinical setting, effective screening methods should be: common, well available, cost-effective, easy to apply, noninvasive, and - foremost - reliable. It is also of importance that the method in question be of high sensitivity and possibly high specificity. In case of the breast tissue, the last two criteria are difficult to attain at a time because the breasts of individual women demonstrate different tissue compositions and densities, hindering thus the final interpretation of the examination. Radiological methods applied so far for the early detection of breast cancer in the general population include ultrasound examination, mammography, and magnetic resonance imaging. Each of these methods has some advantages and shortcomings. Whereas ultrasound is entirely safe for the patient and offers a rather good insight into the breast of mainly glandular composition, mammography is a routine method for the detection of lesions in breasts of predominant adipose composition, however, at the expense of patient exposition to ionizing radiation.

Magnetic resonance imaging demonstrates the best sensitivity and specificity of the three, yet high costs of the examination and the necessity to give the patient a contrast medium limit its broader application for in-depth diagnosis. Therefore, new and 
complementary methods are constantly being invented and tested for the early detection of breast cancer in hope that these inventions will turn suitable for screening and will thus save more human lives. The aim of the present communication is to report the first set of data obtained with a novel instrument designed to examine the breasts, hybrid ultrasound tomography scanner by DRAMINSKI Ultrasound Scanners Company, (Olsztyn, Poland), as a new means of cancer screening.

\section{Materials and Methods}

\section{Description of Ultrasound Tomography Scanner}

The ultrasound tomography is noninvasive and painless, as there is neither mechanical compression of the breast, nor ionizing radiation applied. During the measurement, patients are placed on the scanner bed in the prone position with the breast submerged in the container filled with water (Figure 1). The breast is scanned in coronal sections in $1 \mathrm{~mm}$ distances, using the ultrasonic circular array shifted vertically [1]. The ring array contains 1024 miniature piezoceramic ultrasonic transducers arranged evenly on the inner side of the ring surrounding the breast. Elementary transducers have active surface dimensions of $0.5 \times 18 \mathrm{~mm}$ and work at a frequency of about $2 \mathrm{MHz}$ [2]. Depending on the length of the breast, about 100 to 200 coronal sections are measured, from its base to the nipple. The Figure 2 shows the block scheme of the hybrid ultrasound tomography scanner (Figures 1 and 2). In ultrasound tomography 3 complementary ultrasound images, representing a distribution of transmission, reflection and scattering ultrasound parameters are reconstructed. The fusion of these images allows a qualitative and quantitative characterization of breast tissue in the entire breast volume [3] (Figure 3).

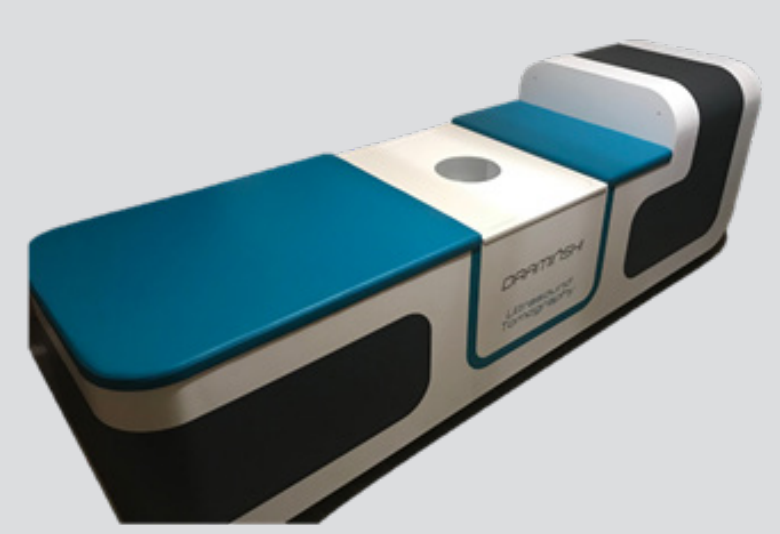

Figure 1: Hybrid ultrasound tomography scanner by DRAMINSKI.

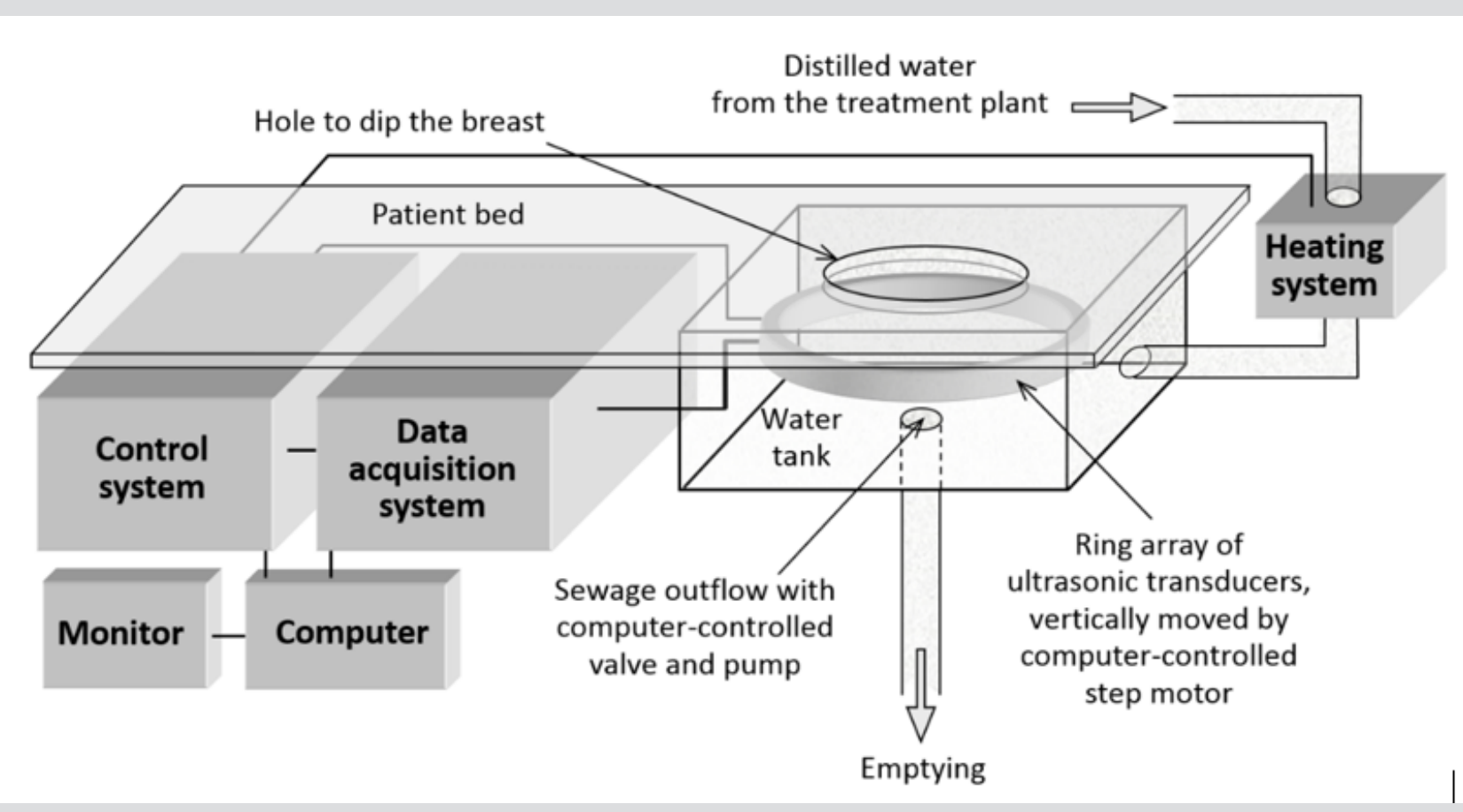

Figure 2: Hybrid ultrasound tomography scanner: a general block scheme. 

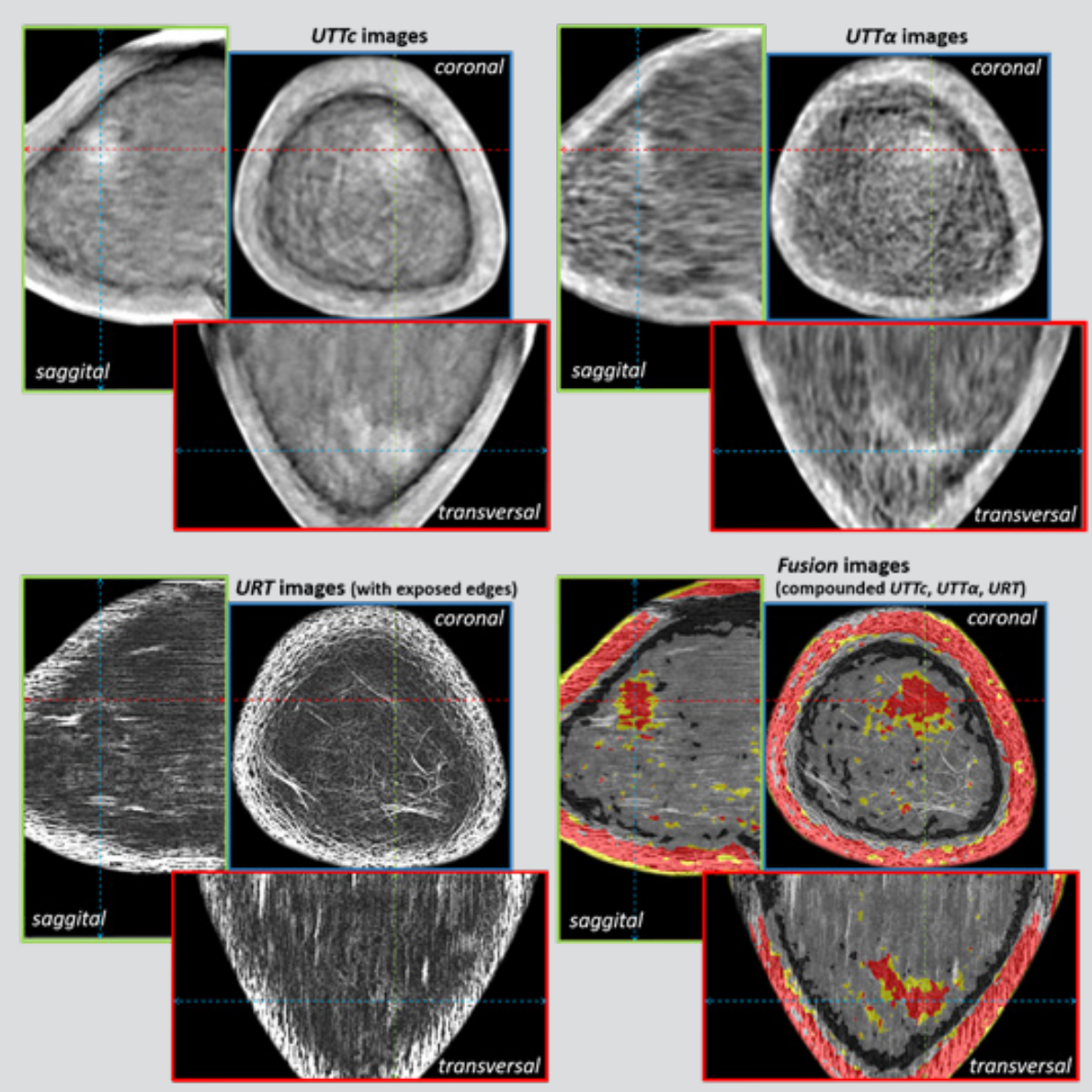

Figure 3: Ultrasound tomography images of the left breast sections of 71 year-old woman with the invasive lobular carcinoma in a large dispersion. Diagnosis has been confirmed by histopathology. Left-up - transmission quantitative images of ultrasound speed distribution; right-up - transmission quantitative-qualitative images of the ultrasound attenuation distribution; leftdown - reflection qualitative images of the ultrasound relative backscattering coefficient distribution; right-down - fusion compounded images.

\section{Patients and Breast Evaluation}

In this study 132 women aged 18-82 years underwent breast examination with the hybrid ultrasound tomography scanner. Each detected lesion of the breast was evaluated on the basis of the red pixel number indicating malignancy in relation to the yellow pixel number indicating benignity. Pixels were counted separately in each coronal, transversal and sagittal section of the lesion in the fusion image (Figure 3), and then averaged. These results were expressed as the "percentage of malignancy" (\%) and taken for the statistical analysis. Breast density was determined according to American College of Radiology [4]. Conventional B-mode ultrasound breast imaging, mammography and magnetic resonance breast imaging results expressed in BIRADS scale were used for comparison.

\section{Results}

Mean patients' age in the study group was 51.8 yrs $( \pm$ SD 14.1 yrs). The analysis showed that young women (mean age 46.12 yrs +/- SD 12.47 yrs) showed more often dense glandular breast tissue (ACR3 and ACR4), whereas older women (mean age 60.04 yrs +/- SD 12.3 yrs) showed less glandular and more fatty breast tissue (ACR 1 and ACR 2), the difference between the groups being statistically significant ( $p<0,000$; Mann-Whitney test). There was a weak, but statistically significant $(\mathrm{p}=0.0018)$ Spearman's rank correlation ( $\mathrm{R}=0.3286)$ of "percentage of malignancy” detected by hybrid ultrasound tomography scanner with ultrasound BIRADS score of the same patients (Figure 4). Similarly there was a weak, significant $(\mathrm{p}=0.0116)$ correlation $(\mathrm{R}=0.3089)$ of „percentage of malignancy" detected by hybrid ultrasound tomography scanner with mammography BIRADS score. No correlation was established between of "percentage of malignancy” and magnetic resonance breast imaging results. Additional analysis showed that the above correlations were present in the group of patients of low breast density (ACR1 and ACR2), but not in patients with glandular breasts. This confirms, that the hybrid ultrasound tomography scanner, similarly to mammography, is more effective in patients of low breast density. The overall sensitivity and specificity of the hybrid ultrasound tomography scanner were computed as $73.9 \%$, and $68.4 \%$, respectively. 


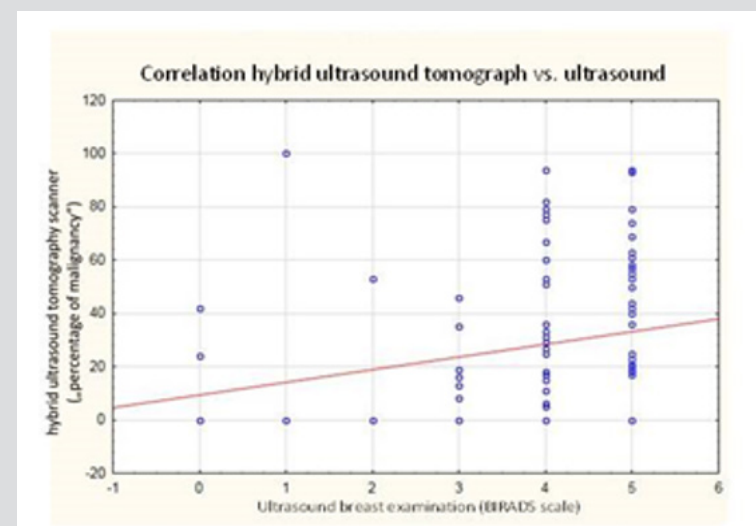

Figure 4: Correlation between hybrid ultrasound tomography expressed as "percentage of malignancy" and breast ultrasound results expressed in BIRADS scale.

\section{Discussion}

Despite great efforts to reduce breast cancer morbidity, breast cancer remains the most frequently diagnosed cancer and the leading cause of cancer-related death among women worldwide [5]. Ultrasound-based methods are promising, as they are noninvasive, and theoretically should have high detection accuracy in younger women. The hybrid ultrasound tomography scanner was concepted as a new tool to improve current radiologic methods of early breast cancer detection. The data form this study deliver information that ultrasound tomography is capable to visualize benign and malignant breast lesions, however, the actual prototype does not match the level of detection comparable to ultrasound or mammography (Table 1). On the other hand, the hybrid ultrasound tomography scanner was able to detect cancer as small as $3 \times 4$ $\mathrm{mm}$, which indicates its excellent potential for early detection and screening. Improvement of scanning algorhythms may help to better distinguish between normal and suspected tissue. These promising initial data warrant further studies on hybrid ultrasound tomography and development of the method [6].

Table 1: Comparison on sensitivity and specificity of breast cancer detection by currently used radiological methods [6] and by hybrid ultrasound tomography scanner (modified).

\begin{tabular}{|c|c|c|}
\hline & sensitivity (\%) & specificity (\%) \\
\hline Ultrasound & 86 & 98 \\
\hline Mammography & 82 & 99 \\
\hline Magnetic resonance (3T) & 100 & 94 \\
\hline Hybrid ultrasound tomography & 74 & 68 \\
\hline
\end{tabular}

\section{Acknowledgment}

Ultrasound breast imaging in vivo has been performed with the approval of the Bioethics Committee at the University of Warmia and Mazury in Olsztyn (Resolution No. 22/2015), within the research titled: "The evaluation of diagnostic value of breast examination using ultrasound transmission tomography and ultrasound reflection tomography". The research was performed as project POIR.01.01.01-00-1595/15, titled: "Development of a prototype of multi-modal ultrasound tomography system for breast diagnosis" co-financed by European Union from the European Regional Development Fund (Sub-task 1.1.1 - Industrial Research and Development Works Conducted by Companies, Smart Growth 2014-2020 Operational Program), as part of the funding granted by the Polish National Center for Research and Development.

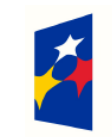

\section{European Funds Smart Growth}

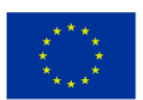

European Union European Regional Development Fund

\section{References}

1. Huy TQ, Tue HH, Ton That Long TT, Duc-Tan T (2017) Deterministic compressive sampling for high-quality image reconstruction of ultrasound tomography. BMC Medical Imaging 17: 34.

2. Opielinski K, Pruchnicki P, Szymanowski P, Szepieniec W, Szweda H, et al. (2018) Multimodal ultrasound computer-assisted tomography: an approach to the recognition of breast lesions. Computerized Medical Imaging and Graphics 65: 102-114.

3. Opielinski K, Pruchnicki P, Gudra T, Podgorski P, Kurcz J, et al. (2015) Imaging results of multi-modal ultrasound computerized tomography system designed for breast diagnosis.Computerized Medical Imaging and Graphics 46: 83-94.

4. Sprague BL, Gangnon RE, Trentham-Dietz A (2014) Prevalence of mammographically dense breasts in the United States. J Natl Cancer Inst pp. 106.

5. Torre LA, Islami F, Siegel RL, Ward EM, Jemal A (2017) Global Cancer in Women: Burden and Trends. Cancer Epidemiology, Biomarkers \& Prevention 26: 444-457.

6. Elsamaloty H, Elzawawi MS, Mohammad S, Herial N (2009) Increasing accuracy of detection of breast cancer with 3-T MRI. AJR Am J Roentgenol 192: 1142-1148. 
ISSN: 2574-1241

DOI: 10.26717.BJSTR.2019.14.002594

Jóźwik Marcin. Biomed J Sci \& Tech Res

(C) (P) This work is licensed under Creative

Submission Link: https://biomedres.us/submit-manuscript.php

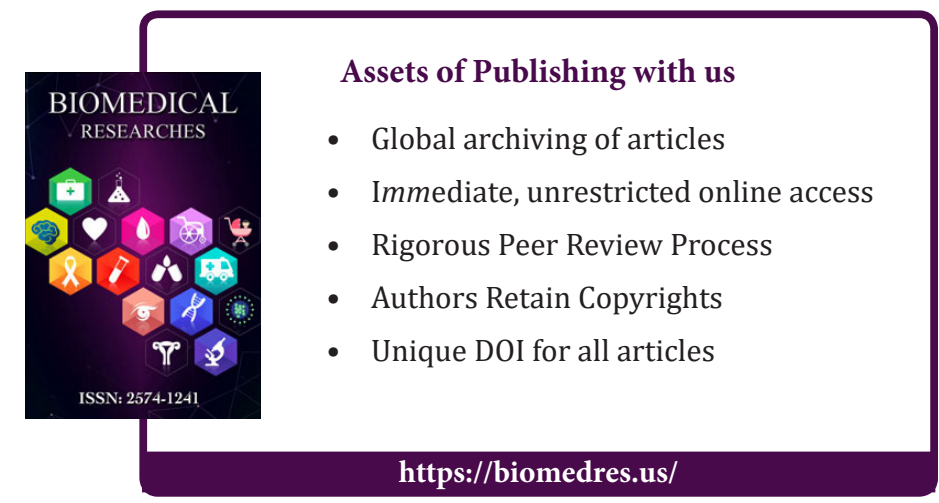

